\title{
Highly Potent Multi-Valent Nanobodies Against Chikungunya with VHH Screened from Alpaca Naïve Phage Display Library
}

Qianlin Li ( $\sim$ moonfesta@126.com)

Sun Yat-Sen University

\section{Fuqiang Zhang}

Center for Disease Control and Prevention of Southern Theater Command Center

Yi Lu

Health Effects Institute

Huan Hu

Sun Yat-Sen University

Jin Wang

Sun Yat-Sen University

Cheng Guo

Columbia University Mailman School of Public Health

Qiang Deng

Sun Yat-Sen University

Conghui Liao

Sun Yat-Sen University

Qin Wu

Sun Yat-Sen University

Tingsong Hu

Center for Disease Control and Prevention of Souther Theater Command

\section{Zeliang Chen}

Sun Yat-Sen University

Jiahai Lu

Sun Yat-Sen University

\section{Research Article}

Keywords: Chikungunya, Nanobody, Naïve Phage Display Library, E2 glycoprotein, Epitopes

Posted Date: February 10th, 2022

DOl: https://doi.org/10.21203/rs.3.rs-1309999/v1 
License: (c) (i) This work is licensed under a Creative Commons Attribution 4.0 International License. Read Full License 


\section{Abstract}

Background $\mathbb{}$ Chikungunya virus (CHIKV) is a re-emerged mosquito-borne alphavirus that can cause musculoskeletal disease and impose a substantial threat to public health globally. It would be desirable to develop a high-affinity antibodies for the diagnosis and therapy of CHIKV infection. As potential diagnostic and therapeutic agents, multivalent nanobodies hold a significant promise towards nanomedicine. Here, we developed the highly potent multivalent nanobodies from an alpaca naïve phage display library targeting the E2 glycoprotein of CHIKV virus. Multivalent nanobodies play an important role in the promotion of high-affinity binding to E2 protein.

Results: In the present study, we generated 20 nanobodies using a naïve phage display library for binders to the CHIKV E2 glycoprotein. Of which, multivalent nanobodies of Nb-2E8 and Nb-3C5 had specific highaffinity binding to E2 protein with nanomolar range, showing the equilibrium dissociation constant (KD) of 2.59-20.7 nM, which is 100-fold stronger than monovalent nanobodies' affinity. Moreover, epitope mapping showed that the Nb-2E8 and Nb-3C5 recognized different linear epitopes located on the E2 glycoprotein domain $C$ and $A$, respectively. A facile protocol of sandwich ELISA was established using the BiNb-2E8 as a capture antibody and HRP-conjugated BiNb-3C5 as a detection antibody. A good linear correlation was achieved between the $O D 450$ value and the E2 protein concentration in the $5-1000 \mathrm{ng} / \mathrm{mL}$ range $(r=0.9864, P<0.0001)$, indicating that it can be used for the quantitative detection of E2 protein.

Conclusions: Multivalent nanobodies Nb-2E8 and Nb-3C5 exhibit functional features and high affinity distinct from monovalent nanobodies, showing new candidate diagnostic applications to detect sera binding protein and/or virions.

\section{Background}

Chikungunya virus (CHIKV) is the causative agent of chikungunya fever (CHIKF), generally causes febrile, arthralgia/arthritis and rash with transmission from person to person mainly by Aedes aegypti and Aedes albopictus mosquitoes. Human CHIKV infection was not reported in Tanzania until 1953 when the pathogen was first major outbreak [1]. In 2007, the World Health Organization (WHO) launched the "Chikungunya Fever, a re-emerging disease in Asia" as a call for an increased focus of worldwide attention on Chikungunya [2]. In the past decades, it is prevalent in Pacific region and Latin America, affects $>100$ countries have been reported in millions of cases $[3,4]$. Recent outbreak occurred in the China-Myanmar border in 2019, which was imported from Myanmar to Ruili City, Yunnan Province, China. This disease is characterized by a low mortality rate, but it could be easily misdiagnosed. Due to the strong infectivity, it is considered to be the rapidly spreading mosquito-borne diseases worldwide following malaria and dengue. While there are no licensed antiviral treatments or vaccines available for CHIKV infection, early diagnosis is nevertheless important.

CHIKV is an enveloped single-stranded RNA Alphavirus [6]. The mature infectious CHIKV is a $\sim 70 \mathrm{~nm}$ icosahedral enveloped particles in diameter, composed of transmembrane glycoproteins E1, E2 and E3. 
E1 and E2 glycoproteins assemble into heterodimers with 80 trimeric spikes of the virions [7, 8]. E1 is a type II transmembrane glycoprotein which mediates fusion of virus and the host cell membrane $[9,10]$. E3 is responsible for the folding of the E2-E1 heterodimer but is cleaved during post-translational maturation [11]. E2 is composed of three immunoglobulin (Ig)-like domains located at the center of an N-terminal domain $A$, the lateral tip of domain $B$, and at the lateral tip of a C-terminal domain $C$. Domain $A$ and domain $B$ is considered to be the binding site of the Mxra8 receptor on the target cell [12]. Because of this structural advantage, CHIKV glycoprotein E2 appears to be to be immunodominant with multiple identified epitopes which induce neutralizing antibodies and has a higher serodiagnostic potential.

VHHs, are single-domain antibodies of molecular weight $\sim 15 \mathrm{kD}$ that are derived from camelid and cartilaginous heavy-chain only antibodies [13,14]. As the smallest functional antibody (nanobody), it is widely studied both in vitro and in vivo and in diagnosis. Compared to conventional antibodies, VHHs are highly stability and solubility, easily purified from E. coli, and typically have nanomolar binding affinities [15-17]. Specifically, the CDR3 of nanobodies plays a key role in recognizing cavities or hidden epitopes such as the virus-binding site of a cell surface receptor [18] and the active site of an enzyme [19, 20]. For fast diagnosis and efficient treatment, screening of nanobodies from naïve library is the most straight forward way. Dong, et al [21] and Huo, et al [22] identified the high-affinity nanobodies against SARS-CoV2 from naïve llama VHH libraries that binds to the S/RBD protein and block its interaction with ACE2. Yan, et al[23] recognized two human procalcitonin(PCT)nanobodies from naïve phage display library and successfully applied to develop a sandwich enzyme-linked immunosorbent assay, which showed a linear working range from $10-1000 \mathrm{ng} / \mathrm{mL}$ of PCT.

Here, we generated 20 nanobodies using a naïve phage display library for binders to the CHIKV E2 glycoprotein. Considering the advantages of activity and affinity, Nb-2E8 and Nb-3C5 were selected as the focus of the present study. Our results suggested that both nanobodies could bind to CHIKV E2 protein and virus particles as well as recognizing different epitopes. In addition, a 100-fold increase in its affinity for the E2 following two nanobodies were further constructed into multivalent formats. It can be used for diagnostic applications to detect sera binding protein and/or virions, either as single VHHs or in combination.

\section{Materials And Methods}

\section{Cell lines and viruses}

Vero cells were obtained from the ATCC and cultured in DMEM supplemented with $10 \%$ FBS (Thermo Fisher Scientific) and $1 \%$ penicillin and streptomycin at $5 \% \mathrm{CO}_{2}, 37^{\circ} \mathrm{C}$.

Chikungunya/human/China/GD134/2010 (GenBank Accession: HQ846359) was isolated from serum of an infected female patient at Guangdong Provincial Center for Disease Control and Prevention. Zika/human/China/GD01/2016 (GenBank Accession: KU740184) was isolated from Guangdong inbound passengers. All experiments involving the CHIKV and ZIKA authentic virus were conducted in Biosafety Level 3 and 2 laboratories, respectively. Virus virions were inactived using $\beta$-propiolactone 
(Sigma-Aldrich) when its concentration was $1: 2000$ at $4{ }^{\circ} \mathrm{C}$ for 24 hours. Complete inactivation of virus was confirmed by the lack of replication in a Vero infection experiment. Supernatants containing virus particles concentrated using PEG-it (SBI Biosciences) overnight at $4{ }^{\circ} \mathrm{C}$ and resuspended in PBS for further use.

\section{Nanobodies screening from naïve phage display library}

A VHH phage display library (AlpaLife) constructed in the vector pADL-10b and containing $\sim 2 \times 10^{9} \mathrm{cfu}$ was inoculated into $2 \times Y$ T supplemented with $100 \mu \mathrm{g} / \mathrm{mL}$ ampicillin $(2 \times Y T A)$ and infected with M13K07 helper phage to obtain a library of $\mathrm{VHH}$-presenting phages. Biopannning of phages displaying $\mathrm{VHHs}$ specific for the Chikungunya/SL-CK1 E2 (Sino Biological) were performed as described previously [24-25]. After the three rounds of panning, 384 individual clones were picked to inoculate $2 \times Y$ TA and were grown overnight at $37^{\circ} \mathrm{C}$, supernatant of clonal phage were detected by phage-ELISA with HRP-conjugated goat anti-M13 IgG antibody (Sino Biological). OD450 values $\geq 0.5$ and $P$ (positive OD450)/N (negative OD450) greater than $3 P / N$ ratios was determined as positive clones. Positive candidates were sequenced (Sangon Biotech) and aligned with complementary determining regions (CDRs) amino acid sequence. Five percent BSA was used as a negative control for each round.

\section{Expression and purification of VHHs in Escherichia coli (E.coli)}

For monovalent nanobodies, sequences were synthesized (Generay Biotech), and subcloned into pETSUMO with a tandem N-terminal His-tag, SUMO-tag, and plasmids were subsequently transformed into $E$. coli BL21(DE3) cells. All protein expression overnight at $18^{\circ} \mathrm{C}$ was induced at $O D 600$ of 0.6 by addition of $0.5 \mathrm{mM}$ IPTG. The His-tagged protein was purified by Talon Metal Affinity Resin (Clontech) according to the manufacturer. The eluates were concentrated using a $1.5 \mathrm{~mL}$ Microsep advance centrifugal devices (PALL) with a molecular weight cut-off of $3 \mathrm{kDa}$. Purity quality was analyzed by Coomassie-stained SDSPAGE.

\section{Expression and purification of Fc conjugated multivalent nanobodies in HEK293 cells}

Bivalent and trivalent VHHs were fused to the Fc region of human IgG1 and cloned into the pcDNA 3.4. Multivalent nanobody units were connected through (GGGGS) $\times 3$ flexible linkers. The Fc-fusion constructs were expressed in HEK 293 cells at $8 \% \mathrm{CO}_{2} 37^{\circ} \mathrm{C}$ for 1 week. Nanobodies in the supernatant were purified using protein A. Purity of all samples was analyzed by SEC-HPLC.

\section{Indirect ELISA to quantitate initial binding}


Microtiter plates (Corning) were coated with $2 \mu \mathrm{g} / \mathrm{mL}$ Chikungunya E2, $10 \mu \mathrm{g} / \mathrm{mL}$ purified virus virions, or the control protein SUMO in carbonate buffer (CBS, pH 9.6) overnight at $4{ }^{\circ} \mathrm{C}$, and blocked with $3 \%$ BSA(Sigma-Aldrich) PBS pH 7.4 at $37^{\circ} \mathrm{C}$. Serial 10-fold dilutions of SUMO-tagged VHHs in $3 \%$ BSA were incubated with the immobilized antigen, followed by incubation with HRP-conjugated goat anti-SMT3 (1:2000, CUSABIO). After wash, $100 \mu \mathrm{L}$ of TMB substrate (TIANGEN) was added the wells and reactions were stopped with $100 \mu \mathrm{L}$ of $1 \mathrm{M} \mathrm{HCl}$. Absorbance was measured at $450 \mathrm{~nm}$ on a Epoch $^{\mathrm{TM}}$ microplate reader (BioTek Instruments Inc., Winooski, VT, USA). EDIII protein of ZIKA was used as the negative control.

\section{Nanobodies were validated by western blotting}

Purified Chikungunya E2 protein $(20 \mu \mathrm{g})$ and purified Chikungunya virion $(50 \mu \mathrm{g})$ were loaded onto $12 \%$ SDS-PAGE and electro-transferred to a PVDF membrane (Millipore). Following blocking of membranes with 5\% BSA in $0.05 \%$ TBST and incubated overnight with 1:500 dilution of nanobodies. Membranes were probed to a SMT3-HRP conjugated antibody (1:1000, CUSABIO) and revelation with SuperSignal West Dura ECL reagent (Thermo Fisher Scientific). Chemiluminescence images were generated with FluorChem E scanner system (ProteinSimpleSan, Jose, CA, USA).

\section{Localized Surface Plasmon Resonance (LSPR) assay}

LSPR measurements were performed using a OpenSPRTM instrument (Nicoyalife) to determine the affinity of monovalent nanobodies to $\mathrm{E} 2$ protein. The $\mathrm{COOH}$ chip (Nicoyalife, Canada) was loaded onto the OpenSPRTM instrument following the standard OpenSPRTM procedure. Run with PBS (pH 7.4) at maximum flow rate $(150 \mu \mathrm{L} / \mathrm{min})$ to reach signal baseline. Sample $200 \mu \mathrm{L}$ of isopropanol and run for 10 $s$ to evacuate air. After baseline is reached, the PBS buffer is rinsed through the sample loop and evacuated with air. Slow down the flow rate of PBS (pH7.4) to $20 \mu \mathrm{L} / \mathrm{min}$, and then load $200 \mu \mathrm{L}$ of $\mathrm{EDC} / \mathrm{NHS}(1: 1)$ solution to activate the $\mathrm{COOH}$ sensor chip. Dilute $200 \mu \mathrm{L}$ of ligand $\mathrm{E} 2$ protein $(0.4 \mathrm{mg} / \mathrm{mL})$ for 4 min and rinse the sample loop with PBS (pH7.4). Sample with $200 \mu \mathrm{L}$ blocking solution, the sample loop is rinsed with PBS and evacuated with air. Baseline was observed for 5 min to ensure stability. Selected nanobodies were diluted into a series of different concentrations and sampled at $20 \mu \mathrm{L} / \mathrm{min}$. Both nanobodies and ligand binding times were $240 \mathrm{~s}$ and natural dissociation was $360 \mathrm{~s}$. Kinetic parameters for the binding reactions were calculated using Trace Drawer software (Ridgeview Instruments $A B)$, One to One analytical model.

\section{ELISA-based Mxra8-Fc binding assay}

A fragment of cDNA encoding the Mouse-Mxra8 extracellular domain (residues 23-336, GenBank accession number NM_024263.4) or Human-Mxra8 extracellular domain (residues 24-337, GenBank accession number NM_032348.3) was appended with a TEV enzyme site and a human IgG1 Fc at the C- 
terminus as well as the IL-2 signal peptide at the N-terminus in pcDNA 3.4 expression vectors and transiently transfected into HEK293 cells followed by media collection and purification using protein A sepharose. Mxra8-Fc binding assay was adapted from previously described [26]. MaxiSorp ELISA plates (Corning) were coated with $2 \mu \mathrm{g} / \mathrm{mL}$ anti-mouse CHKV E2 monoclonal antibodies in CBS (pH 9.6) overnight at $4{ }^{\circ} \mathrm{C}$. Washed four times with PBS and blocked with $4 \%$ BSA(Sigma-Aldrich) for $1 \mathrm{~h}$ at room temperature (RT). CHIKV virions $(1 \mu \mathrm{g} / \mathrm{ml})$ were diluted and added for $1 \mathrm{~h}$ at RT. After washing, MoNb-2E8 and MoNb-3C5 or Mouse Mxra8-Fc fusion protein (all at $10 \mu \mathrm{g} / \mathrm{ml}$ ) were incubated for $30 \mathrm{~min}$. Plates were washed and Human Mxra8-Fc (10-fold serial dilutions) were added to the plates and incubated for $1 \mathrm{~h}$ at RT. Plates were washed again and incubated with secondary Rabbit Anti-Human IgG-Fc (1:5000, Bioss). After washing, the plates were developed with TMB substrate (TIANGEN) and $2 \mathrm{~N} \mathrm{H}_{2} \mathrm{SO}_{4}$. Absorbance was measured at $450 \mathrm{~nm}$.

\section{Indirect immunofluorescence assay}

An indirect immunofluorescence assay was developed on CHIKV-infected Vero cells, as previously described [27]. Briefly, infected-cells ( $\mathrm{MOI}, 1)$ were cultured for $72 \mathrm{~h}$ and then fixed in $4 \%$ paraformaldehyde (Biosharp) for 20min. PFA-fixed cells were permeabilized and blocked with $1 \%$ Triton X-100(MP)/1\% BSA(Sigma-Aldrich)/PBS for $1 \mathrm{~h}$ at room temperature. The cells were incubated with nanobodies $(10 \mu \mathrm{g} / \mathrm{mL})$ at $37^{\circ} \mathrm{C}$ for $30 \mathrm{~min}$, followed by Alexa Fluor 488 Anti-6xHis tag antibody (Abcam) or Cy3labeled Goat Anti-Rabbit IgG $(\mathrm{H}+\mathrm{L})$ (Beyotime) for $30 \mathrm{~min}$ at $37^{\circ} \mathrm{C}$. After washing with PBS, cells were observed under a fluorescence microscope (Mshot). Anti-CHIKV rabbit serum was obtained from a rabbit immunized with $\beta$-propiolactone CHIKV GD134 virion and used as a positive control. Anti-CHIKV E1 mouse IgG antibody (R\&D Systems) was used as a control antibody.

\section{Flow cytometry assay}

The binding of nanobodies to virus on cell surface was assessed by flow cytometry assay. Monolayers of Vero cells were infected with CHIKV and ZIKA at an $\mathrm{MOI}$ of 0.1 and harvested at $48 \mathrm{~h}$ post infection. Cells were then fixed with $4 \%$ paraformaldehyde, and permeabilized with permeabilization buffer $(0.05 \%$ Triton$X$ in PBS). $10 \mu \mathrm{g} / \mathrm{ml}$ nanobodies were added into cells and incubated for $60 \mathrm{~min}$ on ice. Mouse anti-E2 monoclonal antibody was used as a positive control. After washing twice with PBS, cells were stained with 1:1000 diluted goat anti-human H\&L (FITC) or FITC Rabbit polyclonal to $6 \times$ His tag (Abcam) for 45 min and analyzed using flow cytometry (Beckman CytoFlex, Brea, CA, USA).

\section{Human sera samples were detected in a sandwich ELISA}

HRP-coupled bivalent VHHs performed using HRP Conjugation Kit (Abcam) following manufacturer instructions. $2 \mu \mathrm{g} / \mathrm{mL}$ of BiNb-2E8 in CBS (pH9.6) was added per well of a coated 96-Well Plate at $4^{\circ} \mathrm{C}$ overninght. After washing with PBST and blocking with 5\% BSA in PBST for $1 \mathrm{~h}, 100 \mu \mathrm{L}$ of E2 protein 
diluted in a 2-fold dilution series (starting dilution 5 in 10240) or human sera samples (dilution 1:10) were added and incubated at room temperature for $1 \mathrm{~h}$. Wells were washed $3 \times 5 \mathrm{~min}$, and $100 \mu \mathrm{L}$ of HRP labeled BiNb-3C5 $(1 \mu \mathrm{g} / \mathrm{mL})$ was added to each. After $1 \mathrm{~h}$ of incubation, plates were washed 5 times with PBST. TMB was added to the wells for colorimetric development and the absorbance was read at $450 \mathrm{~nm}$. Human Mxra8-hFc served as the control. Samples were considered seropositive if OD450 values higher than the mean obtained for the negative samples plus 3 standard deviations. The CHIKV positive sera samples were sourced from Center for Disease Control and Prevention of Southern Theater Command. Twenty CHIKV negative sera from healthy subjects were used for the calculation and validation of the cut-off value and excluded the sample matrix.

\section{Epitope-binding using peptide-based ELISA}

A pool of 10-mer peptides with 5 amino acid overlap spanning the Chikungunya/SL-CK1 E2 glycoprotein were generated by chemistry to a purity of $90 \%$ (GL Biochem). All peptides were provided as lyophilised power, reconstituted in DMSO to a concentration of $1 \mathrm{mg} / \mathrm{mL}$, and stored at $-80^{\circ} \mathrm{C}$. Each peptide was coated at $1 \mathrm{ug} / \mathrm{mL}$ in $\mathrm{CBS}$ (pH 9.6) overnight at $4{ }^{\circ} \mathrm{C}$ and then blocked with $5 \%$ BSA(Sigma-Aldrich) in PBST. An irrelevant SARS-CoV-2 peptide was used as the negative control. Binding of the coated peptide was characterized by incubation with $2 \mu \mathrm{g} / \mathrm{mL}$ nanobodies. HRP Mouse monoclonal to $6 \times$ His tag (Abcam) used at 1:5000 dilution in blocking buffer, and further absorbance measurement of the enzymatic reaction in TMB substrate (TIANGEN), were used to detect the bound epitopes.

\section{Prediction of the conformation of nanobodies complex with CHIKV E2 protein}

The homology model of protein structure was built on the DeepMind algorithm AlphaFold system (https://deepmind.com/). The complex model of Nb-2E8 or Nb-3C5 to CHIKV E2 protein were genetated by pyDOCK (https://life.bsc.es/pid/pydockweb). All structural data of the docking models were visualized using PyMOL software (https://pymol.org/2/).

\section{Statistical analysis}

All experiments were performed at least two times in duplicate, and representative data or pooled data from repeat experiments were recorded. Statistical analyses were carried out using GraphPad Prism Software (San Diego, CA, USA). Data were presented as the mean \pm SD. Students t-test was used for two groups comparison. Two-sided $P$-values $<0.05$ were considered statistically significant.

\section{Results}




\section{Screening and characterization of nanobodies targeting the E2 Protein}

Nanobodies have become increasingly accessible using high-throughput naïve libraries to rapidly recognize multiple target antigens while resisting adverse biochemical properties such as instability, multi-reactivity and aggregation during affinity maturation [28, 29]. To obtain active protein-binding clones from enriched library, we conducted three rounds of screening (Fig. 1a) and examined for specific protein-binding using a phage ELISA. Table S1 showed that the phage clones specific to the CHIKV E2 protein were effectively enriched through consecutive selection rounds. A total of 119 positive clones were included for the genomic sequencing and data analyses (Table S2). After complementarity determining regions (CDRs) sequence analysis, 20 unique CHIKV E2 specific nanobodies were identified (Fig. 1b and 1c). These sequences were clustered into several discrete groups based on VHH similarities (Fig. 1d).

\section{Soluble expression and purification of the nanobodies using the E.coli system}

Using the prokaryotic system in E.coli strain BL21, we expressed recombinant nanobodies with Nterminally 6xHis-and SUMO-tagged protein (Fig. 2a). The expressed nanobodies can exist as intracellular soluble and active proteins mostly found in the lysate supernatant (Fig. S1). However, Nb-4C1 and Nb4G10 were aggregated into inclusion bodies in E.coli cytoplasm (Fig. S1). Soluble nanobodies were further purified by cobalt-chelating Sepharose columns using $250 \mathrm{mM}$ imidazole for elution. Coomassiestained SDS-PAGE showed the recombinant proteins were detected at the expected apparent molecular weights, $28 \mathrm{kDa}$ (Fig. 2b). To confirm the specificity of binding of 20 candidate nanobodies, CHIKV E2 as coating antigen were carried out by indirect ELISA (Fig. S2). Of these, Nb-2E8 and Nb-3C5 can specifically bind with E2 protein as well as CHIKV virion (Fig. 2d). Moreover, western blot showing Nb-2E8 and Nb-3C5 were also able to recognize the purified CHIKV virion and E2 protein (Fig. 2C). The results suggest that both nanobodies recognize linear epitopes on CHIKV E2.

\section{Binding and identification profiles for MoNb-2E8 and MoNb- $3 \mathrm{C5}$}

To validate the binding capabilities of nanobodies targeting E2 protein, we measured the binding kinetics of nanobodies by LSPR. While MoNb-2E8 and MoNb-3C5 have an affinity of $101 \mathrm{nM}$ and $368 \mathrm{nM}$ (Fig. 3a), showed a weak affinity with E2 protein. We also evaluate whether Nb-2E8 and Nb-3C5 compete with Mxra8-Fc to bind with CHIKV. Both Human Mxra8-Fc and Mouse Mxra8-Fc could noncompetitively bind to CHIKV (Fig. 3c). This would explain that, Nb-2E8, Nb-3C5 and Mxra8 interact at different sites with virions and most likely do not compete for the same epitope. Nb-2E8, Nb-3C5 and anti-rabbit serum were used as 
primary antibodies to detect the expression of viral E2 protein in Vero cells at $72 \mathrm{~h}$ post-infection. Fig. 3b showed that specific green and red fluorescence from were observed in the virus-infected cells, but not virus-uninfected cells. In order to further confirm the binding specificity of the nanobodies, CHIKV infected cells were labeled with Nb-2E8 and Nb-3C5 and subjected to flow cytometry (Fig. 3d). Blue boxes, the proportion of cells in which the fluorescence value exceeded the gating threshold. Quantifications presented in ratios of fluorescence-positive cells to the total number of cells, revealed that the FITCpositive values of CHIKV infected-cells were visibly higher than ZIKA infected-cells $(P<0.05)$.

\section{Establishment of the sandwich ELISA}

To further improve the affinity of nanobodies against CHIKV E2, Nb-2E8 and Nb-3C5 were fused to an Fc domain of IgG1 to generate multivalent fusion proteins and obtain milligram quantities of highly pure (>95\%) recombinant proteins (Fig. 4a, 4b, and Table S3). BiNb-2E8, BiNb-3C5, TriNb-2E8, and TriNb-3C5 showed high binding affinities to CHIKV E2 with equilibrium dissociation constants (KD) of 2.59, 20.70, 4.52, and 10.40, respectively (Fig. 3a). Cytofluorimetric quantification of BiNb-2E8 stained cells to the total number of cells was $95.81 \%$ and $95.20 \%$ for BiNb-3C5 (Fig. S3). We developed a sandwich ELISA protocol, purified bivalent nanobodies was used as the capture and different conjugated bivalent nanobodies was used as the detection, to quantify relative antigen response levels. From 4 independent experiments, the linear equation of $Y=6.908 \mathrm{e}-004 \mathrm{X}+0.7684(r=0.9864, P<0.0001)$ was found to be the optimal linear fit, which demonstrated good linearity over the concentration range $(5-1250 \mathrm{ng} / \mathrm{mL})$ (Fig. 4c). The BiNb-2E8 was combined as capture and the HRP-labelled BiNb-3C5 as detection to reveal combination with good performance.

\section{Validation with human sera samples}

To establish a baseline for the evaluation of the E2 antigen capture test, $20 \mathrm{CHIKV}$ seronegative samples were analysed. Cutoff was defined as the mean OD450 value of the negative controls + 3 SD: $0.0696+$ $(3 \times 0.0128)=0.108$. Hence, sera samples giving $O D 450$ values higher than 0.108 were recorded as positive, and negative when $O D 450$ values lower than 0.108 . By this criteria, all 15 serum of patients infected with CHIKV had OD values greater than that of the cut-off value, which were judged as positive. Also, the difference between CHIKV seronegative and seropositive samples were highly significant statistically $(P<0.0001)$ (Fig. 4d). Therefore, the sandwich ELISA established in this study can be used in the early diagnosis of CHIKV viral infection.

\section{Mapping linear epitopes of the E2 protein using Nb-2E8 and $\mathrm{Nb}-3 \mathrm{C} 5$}

To characterize specific linear epitopes of CHIKV E2 protein, 68 peptides with an offset of ten amino acids were used to assess responses (Table S4). As shown in Fig. 5b, P61- GEEPNYQEEW and P10- 
LKIQVSLQIG strongly reacted with Nb-2E8 and Nb-3C5, which correspond to the amino acid residues 331 to 340 and 46 to 55 of CHIKV E2 domain C and A, respectively (Fig. 5a). Comparison of epitopes of the $\mathrm{Nb}-2 \mathrm{E} 8$ and $\mathrm{Nb}-3 \mathrm{C} 5$ with sequences from representative strains of each CHIKV genotype (SL-CK1, BR33, CU-Chik683 and Ross: East, Central, and South African (ECSA); RSU1: Asian; Senegal: West African) (Fig. $5 \mathrm{c}$ ). Of note is that $\mathrm{Nb}-3 \mathrm{C} 5$ recognised epitope which is highly conserved across the six-representative $\mathrm{CHIKV}$ genotype. And two amino acids at sites were fairly well conserved among other alphaviruses genus. The Nb-2E8 epitope had two residue variation at positions 302 (E-Q) and $307(\mathrm{Q}-\mathrm{H})$ in West African genotype of CHIKV and was not identical among all representative of alphaviruses. For visualization, computer docking simulation was used to map the binding of nanobodies to the E2 protein. Similar to peptide-based ELISA, the formation of complex demonstrates Nb-2E8 and Nb-3C5 bind different epitopes on E2 (Fig. 5d). In the complex, residues L98, G99, W100, T101, and L108 from CDR3 loop of Nb-2E8 have made contacts with the P61epitopes of E2. The CDR1 and CDR3 region of Nb-3C5 has contributed to the P10 epitopes of domain C.

\section{Discussion}

Nanobody opens important possibilities for biomedical applications, but so far most generated by immunized Camelidae, which is time-consuming and expensive for laboratories. Naïve phage display library, a technique for obtaining genetically engineered nanobodies against an antigen without immunization[30], allow the isolation of antibodies against autoantigens, non-immunogenic or toxic antigens. High affinity antibodies can be obtained in a short time when the library capacity is sufficiently large. $\mathrm{VHH}$, also namely nanobody, is 10 -fold smaller than conventional antibodies with unique advantages[31]. As a result of their small size and stability, it is easily incorporated into the body's natural metabolic pathways. More importantly, VHH domains have longer complementary antigen-binding regions CDR3, providing greater antigen-binding capacity, and the affinity of nanobodies can be further improved by genetic engineering techniques[32]. In this study, we performed genetically engineered nanobodies targeting CHIKV by using phage display technique based on a naïve antibody library derived from 103 healthy adult alpaca lymphocytes with a capacity of $2 \times 10^{9} \mathrm{cfu}$, which was able to screen for specific binding nanobodies. A total of $20 \mathrm{VHH}$ sequences specifically binding CHIKV E2 were obtained, containing CDR1, CDR2 and CDR3. The amino acid sequence length of CDR3 region varies from 17-20, with $85 \%$ of the sequences greater than 17 amino acids, indicating typical characteristics of a nanobody. In the FR2 region, high stability and solubility of $\mathrm{VHH}$, attributing to the typical substitutions of hydrophobic residues. Altogher, nanobody was a convenient monovalent scaffold, with the single chain easily expressed in E. coli.

The pET-SUMO expression system, which combines the pET plasmid with SUMO partner together, linking the target gene to SUMO via homologous recombination cloning strategy, and is efficiently and stably expressed under the control of strong T7 promoter[33]. The SUMO protein was an ideal candidate tag for enhancing expression, folding and stability of proteins[34, 35], especially for heterologous proteins like antibody fragments[36]. Several studies have reported recombinant proteins have been efficiently 
produced using the SUMO fusing system in E. coli [37-39]. The results from this study agree with the previous study. Nanobodies were secreted to the culture medium in a soluble form. An N-terminal $6 \times$ His Tag was added to the express vector to aid purification and, SDS-PAGE analysis revealed a single band at approximately $28 \mathrm{kDa}$. However, two nanobodies $\mathrm{Nb}-4 \mathrm{C} 1$ and $\mathrm{Nb}-4 \mathrm{G} 10$ were expressed in E. coli and refolded from inclusion bodies. We speculated that it can potentially be associated with VHH sequences.

For the purified nanobodies, after identified by ELISA, Western blot, and IFA mediated overlap experiment, $\mathrm{Nb}-2 \mathrm{E} 8$ and Nb-3C5, which we chose for further validation. SPR technology is widely known as a golden standard for capturing antibody-antigen interactions[40,41]. To determine the kinetic rate and affinity constants, binding analysis of E2 and monovalent nanobodies were carried out by LSPR. SPR results demonstrated that the binding affinity of MoNb-2E8 and MoNb-3C5 is a relatively low. Obviously, recombinant fusions of monovalent nanobodies are limited by weak binding affinity due to the lack of multivalent affinity benefits, poor production capacity and potential immunogenicity[42-44]. To further improve the affinity of nanobodies against CHIKV E2, homo-dimer (BiNb-2E8, BiNb-3C5) and homo-trimer (TriNb-2E8, TriNb-3C5) were fused to an Fc domain of IgG1 to generate multivalent fusion proteins. We surprisingly found that compared with the corresponding monovalent nanobodies, both homo-dimer and homo-trimer show a 100-fold enhancement towards E2 protein. Among them, BiNb-2E8 was confirmed by LSPR analysis, with a binding affinity of $\sim 2.59 \mathrm{nM}$. Developing highly affinity and specific nanobodies for capturing viral antigens is crucial for any point-of-care testing (POCT) such as ELISA to be successful. Thus, we capitalize on the strengths of the selected nanobodies to establish a rapid, convenient and reliable screening test.

A major advantage of the ELISA method is that it can detect viruses at a concentration of $1-10 \mu \mathrm{g} / \mathrm{ml}$ and require little antibodies[45]. Traditional antibodies considered for developing ELISA have been polyclonal and monoclonal antibodies. However, these highly specific antibodies are cost-effective and have poor stability under adverse environmental conditions[46]. Nanobodies are powerful tools for a wide application in molecular biology, providing high affinity and antigen specificity. In this study, we prepare specific multivalent nanobodies against E2 protein and develop a double-antibody sandwich ELISA for the detection of antigen quality using BiNb-2E8 as capture antibodies and BiNb-3C5 as detection antibody. A good linear correlation was achieved in the $5-1000 \mathrm{ng} / \mathrm{mL}$ range. ELISA results showed that patients with CHIKV had significantly higher serum E2 levels than CHIKV seronegative $(P<0.0001)$. Furthermore, the best choice for coated antibodies is to recognise only a single antigenic determinant[47-49]. Two types of diagnostic and protective antigens, ZIKA and SARS-CoV-2, were tested by indirect ELISA. Nb-2E8 and Nb-3C5 were found no binding activity to react with ZIKA and SARS-CoV-2 protein without any dose dependent manner. Additionally, flow cytometry analysis the two nanobodies of $\mathrm{Nb}-2 \mathrm{E} 8$ and Nb-3C5 positively stain CHIKV-infected cells but negative on ZIKA-infected cells. Overall, Nb2E8 and Nb-3C5 were specific for CHIKV and had no cross-reactivity with ZIKA and SARS-CoV-2. These findings indicated that the ELISA protocol could be performed in any laboratory and served as the foundation of an inexpensive tool for the early diagnosis of CHIKV. 
An understanding of the interaction between the antibody and its targeted antigen and knowing of the epitopes are critical for developing epitope based diagnostic reagents. The epitopes recognised by the nanobodies were identified by peptide-based ELISA, and the epitopes sites were located. Previous studies have reported that several monoclonal antibodies targeting the structural of domain $A$ and $B$ could block fusion and inhibit interaction with cellular receptor $[8,50]$. Epitope mapping revealed that Nb-2E8 and Nb$3 \mathrm{C} 5$ bound the different linear epitope distributed in domain $\mathrm{C}$ and domain $\mathrm{A}$, respectively. In particular, the P10-46“LKIQVSLQIG"55 has not been reported. But we had previously predicted that the peptide "QVSLQIGIK" was the immunodominant B- and T-cell epitope using in silico techniques (unpublished data). Several studies suggested that the P61-301 "GEEPNYQEEW"310 was found to be B-cell epitopes shared by human and mice $[51,52]$. Unfortunately, the selected two nanobodies that focus on the domains of the target linear epitopes are non-neutralizing. The main neutralization sites on the CHIKV E2 protein are likely to be available in a conformation-dependent manner, rather than as linear epitopes.

Based on sequence alignment analysis, the P10 was completely conserved among all CHIKV genotype strains analyzed here, indicating that the $\mathrm{Nb}-3 \mathrm{C} 5$ are potentially capable of recognising three $\mathrm{CHIKV}$ genotypes (ECSA, Asian, and West African). It also found that the P61 epitope sequence in West African lineage CHIKV strain had two single two amino acid difference from the other genotypes. This may limit the potential diagnostic applications of Nb-2E8 in countries where West African lineage strains of the virus circulate. However, all epitopes had significant amino acid mutations across other alphaviruses (Mayaro virus, O'nyong'nyong virus, Semliki Forest virus, Ross River virus, Sindbis virus, etc.). These features enable the differential diagnosis of CHIKV. Taken together, these nanobodies, Nb-2E8 and Nb$3 \mathrm{C} 5$, specifically recognize different epitopes of the CHIKV E2 protein, which are the optimal pair of nanobodies for the development of a double antibody sandwich ELISA.

There are several potential limitations to the study. First, our study is only focused on highly potent antibodies against CHIKV. Further studies including are need to demonstrated other nanobodies could interact with E2 protein and to evaluate the diagnostic or treatment merit of specific nanobodies. Second, the present study illustrates a strategy to mine the valuable nanobodies by naïve phage display library. The results support the feasibility of nanobodies for a rapid diagnostic tool. However, the diagnostic accuracy parameters and procedures in this study have not been directly optimized. To establish a standardized CHIKV diagnostic process, the optimization of antibody reagents is required. Third, the binding site prediction of nanobody-E2 complex rely on peptide-ELISA and homology-modeling applications, lacking X-ray diffraction (XRD) or cryoelectron microscopy to investigate the crystal structure parameters.

\section{Conclusions}

In summary, we developed nanobodies from naïve phage display library that addresses the specific needs for CHIKV serodiagnosis. The selected nanobodies of Nb-2E8 and Nb-3C5 could bind CHIKV E2 protein with high specificity and affinity in a multivalent platform. These results suggest that the selected 
nanobodies are used for diagnostic applications to detect sera binding protein and/or virions, either as single VHHs or in combination.

\section{Declarations}

\section{Funding}

This work was funded by the National Key Research and Development Program of China (2018YFE0208000), the Key-Area Research and Development Program of Guangdong Province (2018B020241002) and the Key Research and Development Program for the 14th Five-Year Plan from the Ministry of Science and Technology, China (20SWAQX02).

\section{Competing interests}

The authors declare that there are no conflicts of interest.

\section{Ethics statement}

The serum samples of CHIKV patients were obtained from Center for Disease Control and Prevention of Southern Theater Command. All patients have agreed to sharing their serum samples by informed consent, and the study was approved by the ethics committee of Sun-Yet Sen university.

\section{Availability of data and materials}

All the data analyzed throughout this study are included in the article.

\section{Authors' contributions}

QLL participated in the experiments and data analyzes, and drafted the manuscript. HH and QD developed the experimental approaches, generated the majority of the reagents, and conducted preliminary experiments. YL and JW contributed to discussion and reviewed the manuscript. FQZ and TSH contributed to sample and data collection. CG provided support with data analysis and validation. $\mathrm{CHL}$ and QW facilitated the organization and coordination between partners. ZLC conceived the study and designed the experiments. JHL supervised and directed the project to ensure its progress. All authors read and approved the final manuscript.

\section{Acknowledgements}


We gratefully acknowledge the staffs of the AlpaLife for their excellent technical assistance. We also thank shaopei Chen and Pro. Jufang Wang (South China University of Technology) for the kind gift of anti-mouse CHIKV E2 monoclonal antibodies.

\section{Author details}

${ }^{1}$ One Health Center of Excellence for Research and Training, School of Public Health, Sun Yat-sen University, Guangzhou 510080, China; ${ }^{2}$ NMPA Key Laboratory for Quality Monitoring and Evaluation of Vaccines and Biological Products, Guangzhou 510080, China; ${ }^{3}$ Key Laboratory of Tropical Diseases Control, Sun Yat-Sen University, Ministry of Education, Guangzhou 510080, China; ${ }^{4}$ Center for Disease Control and Prevention of Southern Theater Command, Guangzhou 510060, P. R. China; ${ }^{5}$ Health Effects Institute, Boston, 02169, United States; ${ }^{6}$ Center for Infection and Immunity, Mailman School of Public Health, Columbia University, New York, NY, United States.

\section{References}

1. Couderc T, Chrétien F, Schilte C, Disson O, Brigitte M, Guivel-Benhassine F, Touret Y, Barau G, Cayet N, Schuffenecker I, Desprès P, Arenzana-Seisdedos F, Michault A, Albert ML, Lecuit M. A mouse model for Chikungunya: young age and inefficient type-l interferon signaling are risk factors for severe disease. PLoS Pathog. 2008;4(2):e29.

2. Burt FJ, Rolph MS, Rulli NE, Mahalingam S, Heise MT. Chikungunya: a re-emerging virus. Lancet. 2012;379(9816):662-71.

3. Joshi P, Yadav P, Mourya D, Sahare L, Ukey M, Khedekar R, Patil D, Barde PV. Laboratory surveillance of chikungunya in Madhya Pradesh, India (2016-2017). Indian J Med Res. 2020;151(1):87-92.

4. Nunes MR, Faria NR, de Vasconcelos JM, Golding N, Kraemer MU, de Oliveira LF, Azevedo Rdo S, da Silva DE, da Silva EV, da Silva SP, Carvalho VL, Coelho GE, Cruz AC, Rodrigues SG, Vianez JL Jr, Nunes BT, Cardoso JF, Tesh RB, Hay SI, Pybus OG, Vasconcelos PF. Emergence and potential for spread of Chikungunya virus in Brazil. BMC Med. 2015;13:102.

5. Roth A, Mercier A, Lepers C, Hoy D, Duituturaga S, Benyon E, Guillaumot L, Souares Y. Concurrent outbreaks of dengue, chikungunya and Zika virus infections - an unprecedented epidemic wave of mosquito-borne viruses in the Pacific 2012-2014. Euro Surveill. 2014;19(41):20929.

6. Gérardin P, Barau G, Michault A, Bintner M, Randrianaivo H, Choker G, Lenglet $Y$, Touret $Y$, Bouveret A, Grivard P, Le Roux K, Blanc S, Schuffenecker I, Couderc T, Arenzana-Seisdedos F, Lecuit M, Robillard PY. Multidisciplinary prospective study of mother-to-child chikungunya virus infections on the island of La Réunion. PLoS Med. 2008;5(3):e60.

7. Metz SW, Gardner J, Geertsema C, Le TT, Goh L, Vlak JM, Suhrbier A, Pijlman GP. Effective chikungunya virus-like particle vaccine produced in insect cells. PLoS Negl Trop Dis. 2013;7(3):e2124. 
8. Sun S, Xiang Y, Akahata W, Holdaway H, Pal P, Zhang X, Diamond MS, Nabel GJ, Rossmann MG. Structural analyses at pseudo atomic resolution of Chikungunya virus and antibodies show mechanisms of neutralization. Elife. 2013;2:e00435.

9. Cotmore SF, Agbandje-McKenna M, Canuti M, Chiorini JA, Eis-Hubinger AM, Hughes J, Mietzsch M, Modha S, Ogliastro M, Pénzes JJ, Pintel DJ, Qiu J, Soderlund-Venermo M, Tattersall P, Tijssen P, Ictv Report Consortium. ICTV Virus Taxonomy Profile: Parvoviridae. J Gen Virol. 2019;100(3):367-368.

10. Voss JE, Vaney MC, Duquerroy S, Vonrhein C, Girard-Blanc C, Crublet E, Thompson A, Bricogne G, Rey FA. Glycoprotein organization of Chikungunya virus particles revealed by X-ray crystallography. Nature. 2010 Dec 2;468(7324):709-12.

11. Kim AS, Zimmerman O, Fox JM, Nelson CA, Basore K, Zhang R, Durnell L, Desai C, Bullock C, Deem SL, Oppenheimer J, Shapiro B, Wang T, Cherry S, Coyne CB, Handley SA, Landis MJ, Fremont DH, Diamond MS. An Evolutionary Insertion in the Mxra8 Receptor-Binding Site Confers Resistance to Alphavirus Infection and Pathogenesis. Cell Host Microbe. 2020;27(3):428-440.e9.

12. Zhang R, Kim AS, Fox JM, Nair S, Basore K, Klimstra WB, Rimkunas R, Fong RH, Lin H, Poddar S, Crowe JE Jr, Doranz BJ, Fremont DH, Diamond MS. Mxra8 is a receptor for multiple arthritogenic alphaviruses. Nature. 2018;557(7706):570-574.

13. Hamers-Casterman C, Atarhouch T, Muyldermans S, Robinson G, Hamers C, Songa EB, Bendahman N, Hamers R. Naturally occurring antibodies devoid of light chains. Nature. 1993;363(6428):446-8.

14. Huang NJ, Pishesha N, Mukherjee J, Zhang S, Deshycka R, Sudaryo V, Dong M, Shoemaker CB, Lodish HF. Genetically engineered red cells expressing single domain camelid antibodies confer longterm protection against botulinum neurotoxin. Nat Commun. 2017;8(1):423.

15. Nordeen SA, Andersen KR, Knockenhauer KE, Ingram JR, Ploegh HL, Schwartz TU. A nanobody suite for yeast scaffold nucleoporins provides details of the nuclear pore complex structure. Nat Commun. 2020;11(1):6179.

16. Ma H, Zeng W, Meng X, Huang X, Yang Y, Zhao D, Zhou P, Wang X, Zhao C, Sun Y, Wang P, Ou H, Hu X, Xiang Y, Jin T. Potent Neutralization of SARS-CoV-2 by Hetero-bivalent Alpaca Nanobodies Targeting the Spike Receptor-Binding Domain. J Virol. 2021;95(10):e02438-20.

17. Ingram JR, Schmidt FI, Ploegh HL. Exploiting Nanobodies' Singular Traits. Annu Rev Immunol. 2018;36:695-715.

18. Detalle L, Stohr T, Palomo C, Piedra PA, Gilbert BE, Mas V, Millar A, Power UF, Stortelers C, Allosery K, Melero JA, Depla E. Generation and Characterization of ALX-0171, a Potent Novel Therapeutic Nanobody for the Treatment of Respiratory Syncytial Virus Infection. Antimicrob Agents Chemother. 2015;60(1):6-13.

19. Crasson O, Rhazi N, Jacquin O, Freichels A, Jérôme C, Ruth N, Galleni M, Filée P, Vandevenne M. Enzymatic functionalization of a nanobody using protein insertion technology. Protein Eng Des Sel. 2015;28(10):451-60.

20. De Genst E, Chan PH, Pardon E, Hsu SD, Kumita JR, Christodoulou J, Menzer L, Chirgadze DY, Robinson CV, Muyldermans S, Matagne A, Wyns L, Dobson CM, Dumoulin M. A nanobody binding to 
non-amyloidogenic regions of the protein human lysozyme enhances partial unfolding but inhibits amyloid fibril formation. J Phys Chem B. 2013;117(42):13245-13258.

21. Dong J, Huang B, Jia Z, Wang B, Gallolu Kankanamalage S, Titong A, Liu Y. Development of multispecific humanized llama antibodies blocking SARS-CoV-2/ACE2 interaction with high affinity and avidity. Emerg Microbes Infect. 2020;9(1):1034-1036.

22. Huo J, Le Bas A, Ruza RR, Duyvesteyn HME, Mikolajek H, Malinauskas T, Tan TK, Rijal P, Dumoux M, Ward PN, Ren J, Zhou D, Harrison PJ, Weckener M, Clare DK, Vogirala VK, Radecke J, Moynié L, Zhao Y, Gilbert-Jaramillo J, Knight ML, Tree JA, Buttigieg KR, Coombes N, Elmore MJ, Carroll MW, Carrique L, Shah PNM, James W, Townsend AR, Stuart DI, Owens RJ, Naismith JH. Neutralizing nanobodies bind SARS-CoV-2 spike RBD and block interaction with ACE2. Nat Struct Mol Biol. 2020;27(9):846854.

23. Yan J, Wang P, Zhu M, Li G, Romão E, Xiong S, Wan Y. Characterization and applications of Nanobodies against human procalcitonin selected from a novel naïve Nanobody phage display library. J Nanobiotechnology. 2015;13:33.

24. Schmidt Fl, Lu A, Chen JW, Ruan J, Tang C, Wu H, Ploegh HL. A single domain antibody fragment that recognizes the adaptor ASC defines the role of ASC domains in inflammasome assembly. J Exp Med. 2016;213(5):771-90.

25. Lu Q, Zhang Z, Li H, Zhong K, Zhao Q, Wang Z, Wu Z, Yang D, Sun S, Yang N, Zheng M, Chen Q, Long C, Guo W, Yang H, Nie C, Tong A. Development of multivalent nanobodies blocking SARS-CoV-2 infection by targeting RBD of spike protein. J Nanobiotechnology. 2021;19(1):33.

26. Zhou QF, Fox JM, Earnest JT, Ng TS, Kim AS, Fibriansah G, Kostyuchenko VA, Shi J, Shu B, Diamond MS, Lok SM. Structural basis of Chikungunya virus inhibition by monoclonal antibodies. Proc Natl Acad Sci U S A. 2020;117(44):27637-27645.

27. Tumkosit U, Siripanyaphinyo U, Takeda N, Tsuji M, Maeda Y, Ruchusatsawat K, Shioda T, Mizushima $\mathrm{H}$, Chetanachan P, Wongjaroen P, Matsuura Y, Tatsumi M, Tanaka A. Anti-Chikungunya Virus Monoclonal Antibody That Inhibits Viral Fusion and Release. J Virol. 2020;94(19):e00252-20.

28. Muyldermans S. Nanobodies: natural single-domain antibodies. Annu Rev Biochem. 2013;82:775-97.

29. Shin JE, Riesselman AJ, Kollasch AW, McMahon C, Simon E, Sander C, Manglik A, Kruse AC, Marks DS. Protein design and variant prediction using autoregressive generative models. Nat Commun. 2021;12(1):2403.

30. Lee CV, Liang WC, Dennis MS, Eigenbrot C, Sidhu SS, Fuh G. High-affinity human antibodies from phage-displayed synthetic Fab libraries with a single framework scaffold. J Mol Biol. 2004;340(5):1073-93.

31. Eden T, Menzel S, Wesolowski J, Bergmann P, Nissen M, Dubberke G, Seyfried F, Albrecht B, Haag F, Koch-Nolte F. A cDNA Immunization Strategy to Generate Nanobodies against Membrane Proteins in Native Conformation. Front Immunol. 2018;8:1989.

32. Muyldermans S, Atarhouch T, Saldanha J, Barbosa JA, Hamers R. Sequence and structure of VH domain from naturally occurring camel heavy chain immunoglobulins lacking light chains. Protein 
Eng. 1994;7(9):1129-35.

33. Butt TR, Edavettal SC, Hall JP, Mattern MR. SUMO fusion technology for difficult-to-express proteins. Protein Expr Purif. 2005;43(1):1-9.

34. Panavas T, Sanders C, Butt TR. SUMO fusion technology for enhanced protein production in prokaryotic and eukaryotic expression systems. Methods Mol Biol. 2009;497:303-17.

35. Malakhov MP, Mattern MR, Malakhova OA, Drinker M, Weeks SD, Butt TR. SUMO fusions and SUMOspecific protease for efficient expression and purification of proteins. J Struct Funct Genomics. 2004;5(1-2):75-86.

36. Ye T, Lin Z, Lei H. High-level expression and characterization of an anti-VEGF165 single-chain variable fragment (scFv) by small ubiquitin-related modifier fusion in Escherichia coli. Appl Microbiol Biotechnol. 2008;81(2):311-7.

37. Bommarius B, Jenssen $H$, Elliott M, Kindrachuk J, Pasupuleti M, Gieren H, Jaeger KE, Hancock RE, Kalman D. Cost-effective expression and purification of antimicrobial and host defense peptides inEscherichia coli. Peptides. 2010;31(11):1957-65.

38. Liu X, Chen Y, Wu X, Li H, Jiang C, Tian H, Tang L, Wang D, Yu T, Li X. SUMO fusion system facilitates soluble expression and high production of bioactive human fibroblast growth factor 23 (FGF23). Appl Microbiol Biotechnol. 2012;96(1):103-11.

39. González AS, Guimarães Assmann AL, Romero Ramos CR, Quelopana MM, Aleixo Silva AC, ThomazSoccol V. Recombinant mutagenic $3 A B C$ protein and monoclonal antibody for quality-control testing in foot-and-mouth disease vaccines. Antiviral Res. 2018;157:93-101.

40. Chi X, Liu X, Wang C, Zhang X, Li X, Hou J, Ren L, Jin Q, Wang J, Yang W. Humanized single domain antibodies neutralize SARS-CoV-2 by targeting the spike receptor binding domain. Nat Commun. 2020;11(1):4528.

41. Spodzieja M, Kuncewicz K, Sieradzan A, Karczyńska A, Iwaszkiewicz J, Cesson V, Węgrzyn K, Zhukov I, Maszota-Zieleniak M, Michielin O, Speiser DE, Zoete V, Derré L, Rodziewicz-Motowidło S. DisulfideLinked Peptides for Blocking BTLA/HVEM Binding. Int J Mol Sci. 2020;21(2):636.

42. Holliger P, Hudson PJ. Engineered antibody fragments and the rise of single domains. Nat Biotechnol. 2005;23(9):1126-36.

43. Attarwala H. Role of antibodies in cancer targeting. J. Nat. Sci. Biol. Med. 2010;1:53-56.

44. Farhadi SA, Bracho-Sanchez E, Fettis MM, Seroski DT, Freeman SL, Restuccia A, Keselowsky BG, Hudalla GA. Locally anchoring enzymes to tissues via extracellular glycan recognition. Nat Commun. 2018;9(1):4943.

45. Hartati L, Bakti D, Tantawi AR, Lisnawita. Detection of virus causes papaya ringspot virus-With the DAS-Elisa (Double Antibody Sandwich-Enzyme-Linked Immunosorbent Assay) method at different levels in North Sumatra. Earth Environ. Sci. 2020;454: 012182.

46. Wang C, Gu B, Liu Q, Pang Y, Xiao R, Wang S. Combined use of vancomycin-modified Ag-coated magnetic nanoparticles and secondary enhanced nanoparticles for rapid surface-enhanced Raman scattering detection of bacteria. Int J Nanomedicine. 2018;13:1159-1178. 
47. Fan B, Sun J, Zhu L, Zhou J, Zhao Y, Yu Z, Sun B, Guo R, He K, Li B. Development of a Novel Double Antibody Sandwich Quantitative Enzyme-Linked Immunosorbent Assay for Detection of Porcine Epidemic Diarrhea Virus Antigen. Front Vet Sci. 2020;7:540248.

48. Choi SY, Rhie GE, Jeon JH. Development of a double-antibody sandwich ELISA for sensitive detection of Yersinia pestis. Microbiol Immunol. 2020;64(1):72-75.

49. Wang J, Zhang S, Ni W, Zhai X, Xie F, Yuan H, Gao S, Tai G. Development and application of a doubleantibody sandwich ELISA kit for the detection of serum MUC1 in lung cancer patients. Cancer Biomark. 2016;17(4):369-376.

50. Pal P, Dowd KA, Brien JD, Edeling MA, Gorlatov S, Johnson S, Lee I, Akahata W, Nabel GJ, Richter MK, Smit JM, Fremont DH, Pierson TC, Heise MT, Diamond MS. Development of a highly protective combination monoclonal antibody therapy against Chikungunya virus. PLoS Pathog. 2013;9(4):e1003312.

51. Lum FM, Teo TH, Lee WW, Kam YW, Rénia L, Ng LF. An essential role of antibodies in the control of Chikungunya virus infection. J Immunol. 2013;190(12):6295-302.

52. Chua CL, Chan YF, Sam IC. Characterisation of mouse monoclonal antibodies targeting linear epitopes on Chikungunya virus E2 glycoprotein. J Virol Methods. 2014;195:126-33.

\section{Figures}

\section{Figure 1}

Screening the CHIKV E2 specific nanobodies from a VHH phage display library. a Biopanning procedure of candidate $\mathrm{VHH}$ domain. b Reactions from the 20 clones specifically binding with CHIKV E2 protein. c Alignment of the amino acid sequences of the isolated anti-CHIKV E2 VHH nanobodies. $\mathbf{d}$ Overall similarity of VHH regions anti-CHIKV E2 nanobody sequences.

\section{Figure 2}

Expression, purification and binding activity detection of nanobodies. a Schematic representation of the expression vector pET-SUMO for nanobody. b Soluble prokaryotic expression and purification of recombinant nanobody using an N-terminally 6xHis- and SUMO-tagged protein ( 28kDa).c Binding of SUMO-tagged VHHs to immobilized CHIKV E2 ( 40kD) or Chikungunya virion was evaluated by western blot. d Binding of SUMO-tagged VHHs to immobilized CHIKV E2 or CHIKV virus was quantified by indirect ELISA. 


\section{Figure 3}

Biophysics of the nanobodies binding to E2. a Binding kinetic for nanobodies were obtained by LSPR. $\mathbf{b}$ CHIKV E2 protein expression in infected cells was analyzed by immunofluorescence staining using $\mathrm{Nb}$ $2 \mathrm{E} 8$ and $\mathrm{Nb}-3 \mathrm{C} 5$. c The abilities of Nb-2E8 and Nb-3C5 to block CHIKV E2 interaction with Mxra8 were determined by a competition ELISA. $\mathbf{d}$ Cell binding of the nanobodies were quantified by flow cytometry. Left: representative flow cytometry plots; right: bars show mean \pm S.E.M.

\section{Figure 4}

Features and applications of multivalent nanobodies. a A schematic of multivalent nanobodies. b SDSPAGE of multivalent nanobodies under non-reducing condition. $\mathbf{c}$ Establishment of standard curve using HRP-labeled bivalent nanobodies. $\mathbf{d}$ Validation of the sandwich ELISA using CHIKV-positive serum samples.

\section{Figure 5}

Distribution profiles of components of E2-Nb complexes and their corresponding epitopes. a Schematic diagram of domains and positions of the CHIKV E2 Glycoprotein. b Potential binding peptides response to the $\mathrm{Nb}-2 \mathrm{E} 8$ and $\mathrm{Nb}-3 \mathrm{C} 5$ as measured by a peptide-ELISA. c Alignment of the Nb-2E8 and Nb-3C5 epitope region with $\mathrm{E} 2$ sequences of different genotype strains and alphaviruses. d Structure docking model of Nb-2E8 and Nb-3C5 bound to CHIKV E2 protein.

\section{Supplementary Files}

This is a list of supplementary files associated with this preprint. Click to download.

- GraphicalAbstract.tiff

- SupplementaryFigure.docx

- SupplementaryTable.xlsx 